\title{
Combination of BCL-2 and MYC protein expression improves high-risk stratification in diffuse large B-cell lymphoma
}

This article was published in the following Dove Press journal:

OncoTargets and Therapy

18 September 2015

Number of times this article has been viewed

\author{
Jing Wang* \\ Min Zhou* \\ Jing-Yan $\mathrm{Xu}^{*}$ \\ Bing Chen \\ Jian Ouyang
}

Department of Hematology, The Affiliated Drum Tower Hospital of Nanjing University Medical School, Nanjing, Jiangsu, People's Republic of China

*These authors contributed equally to this work and should be considered as cofirst authors
Correspondence: Bing Chen Department of Hematology, The Affiliated Drum Tower Hospital of Nanjing University Medical School, Nanjing, Jiangsu, People's Republic of China

Tel +862583106666 Ext. 6144I

Fax +86 2583105211

Email chenbing2004@।26.com
Purpose: To evaluate whether the addition of two biological markers (MYC and BCL-2 protein overexpression) improves the stratification of high-risk patients with diffuse large B-cell lymphoma (DLBCL).

Method: Seven risk factors were identified at diagnosis, and a maximum of 7 points were assigned to each patient. The patients were classified according to four risk groups: low (0-1), low-intermediate (2-3), high-intermediate (4), and high (5-7). Only high-risk patients with DLBCL were included in this analysis. We retrospectively examined 20 cases from 2008 to 2013 at the Nanjing Drum Tower Hospital.

Results: The median expression of MYC protein was $60 \%$, and 17 of 20 (65\%) evaluable cases overexpressed MYC. The median expression of BCL-2 protein was also $60 \%$. Eighteen of $20(90 \%)$ evaluable cases showed BCL-2 overexpression. Additionally, 12 out of 20 cases $(60 \%)$ demonstrated coexpression of MYC and BCL-2 proteins. The percentages of overall survival and progression-free survival at the median follow-up time (36 months) were $33.3 \% \pm 16.1 \%$ and $16.9 \% \pm 13.5 \%$, respectively. By comparison, nine, four, and 20 patients were classified as high risk based on the International Prognostic Index (IPI), National Comprehensive Cancer Network(NCCN)-IPI, and revised IPI criteria, respectively. According to the IPI and NCCN-IPI stratification, the risk groups demonstrated closely overlapping survival curves. In addition, four out of 20 cases were identified as low-intermediate risk according to the NCCN-IPI criteria.

Conclusion: The addition of MYC and BCL-2 protein expression to the IPI could identify a subset of DLBCL patients with high-risk clinicopathological characteristics and poor clinical outcome.

Keywords: diffuse large B-cell lymphoma, MYC, BCL-2, International Prognostic Index

\section{Introduction}

Diffuse large B-cell lymphoma (DLBCL) is a heterogeneous group of B-cell lymphomas with various immunophenotypes, different molecular and genetic abnormalities, and a wide range of clinical presentations and outcomes. ${ }^{1,2}$ The International Prognostic Index (IPI) has been the primary clinical tool used to predict the prognoses of patients with DLBCL. ${ }^{3}$ Its predictive capacity has been validated in the past two decades based on the clinical characteristics of patients treated with CHOP-like chemotherapy. The introduction of the anti-CD20 monoclonal antibody rituximab in chemotherapies has transformed the treatment practices for aggressive lymphoma and led to a marked improvement in outcome. ${ }^{4,5}$ As a result, the capacity of the IPI to discriminate between risk groups has declined in the era of immunochemotherapy, particularly among the high-risk patients. ${ }^{6-8}$ An increasing number of studies have indicated that $\mathrm{MYC}$ and/or 
BCL-2 protein detected by immunohistochemistry could be used to identify distinct outcomes of patients with DLBCL. ${ }^{9-14}$ We have also created a novel clinicopathologic prognostic model to predict the prognoses of DLBCL patients. ${ }^{10}$ On the basis of the combination of MYC expression and IPI at the time of diagnosis, three discrete outcome groups have been identified with a 3 -year overall survival (OS), ranging from $39.1 \%$ in the high-risk group to $100 \%$ in the low-risk group. ${ }^{10}$ Most studies agree that patients with DLBCL expressing both MYC and BCL-2 proteins have an aggressive clinical course and a poor outcome..$^{11,15-17}$ The addition of the biological markers MYC and BCL-2 to the IPI might enhance its capacity to prognosticate. ${ }^{18}$ The aim of this study was to evaluate whether the addition of the biological markers MYC and BCL-2 protein overexpression could improve the stratification of high-risk patients with DLBCL, compared with the IPI, NCCN-IPI, ${ }^{19}$ and revised IPI alone. ${ }^{7}$

\section{Materials and methods Patient selection}

This study is a retrospective analysis of patients with de novo DLBCL. Seven risk factors (age $>60$ years, stage III/IV disease, elevated lactate dehydrogenase level, Eastern Cooperative Oncology Group [ECOG] performance status $\geq 2$, more than one extranodal site of disease, high MYC expression, and high BCL-2 expression) of patients at diagnosis were identified, and a maximum of 7 points were assigned to each patient. The patients were classified according to four risk groups: low (0-1), low-intermediate (2-3), high-intermediate (4), and high (5-7). Only high-risk patients with DLBCL were included in this analysis. We retrospectively examined 20 cases from 2008 to 2013 at the Nanjing Drum Tower Hospital. All cases were diagnosed according to the World Health Organization classification criteria. All patients were treated with an R-CHOP regimen. This study was approved by the institutional review board, and all patients gave written informed consent.

\section{Immunohistochemistry}

FFPE sections of $3 \mu \mathrm{m}$ were placed on adhesive-coated slides. Immunohistochemistry for BCL-2 and MYC protein expression was performed as previously reported. ${ }^{10,11}$ Cutoff values of $30 \%$ for BCL-2 staining and $50 \%$ for MYC staining were considered to be high. ${ }^{10,11}$

\section{Statistical analysis}

This analysis is based on patient follow-up data obtained through to August 5, 2014. Progression-free survival (PFS) was calculated from the date of diagnosis to the date of documented disease progression; observations were censored on the date the patient was last known to be alive or for patients dying as a result of causes unrelated to lymphoma or treatment. OS was calculated from the date of diagnosis until death as a result of any cause or the date last known alive. PFS and OS were assessed using the Kaplan-Meier method and compared among risk groups using the log-rank test. The data were analyzed using SPSS version 11.0.

\section{Results}

\section{Patients}

A summary of the major clinical characteristics of the patients included in this study is listed in Table 1 . The median age was 63 years old (range: 30-75 years). The expression of MYC and BCL-2 proteins was quantified according to previously published cutoff values. ${ }^{10,11}$ The median expression of MYC protein was $60 \%$ and 17 out of $20(65 \%)$ evaluable cases overexpressed MYC (Figure S1A). The median expression of BCL-2 protein was $60 \%$. Eighteen out of $20(90 \%)$ evaluable cases showed BCL-2 overexpression (Figure S1B). Additionally, 12 out of 20 cases (60\%) had coexpression of MYC and BCL-2 proteins.

\section{Outcome according to standard IPI, NCCN-IPI, and revised IPI}

The percentages of OS and PFS at the median follow-up time (36 months) were $33.3 \% \pm 16.1 \%$ and $16.9 \% \pm 13.5 \%$, respectively. The median survival time for OS was $24.0 \pm 15.49$

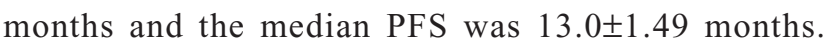
The patient outcomes according to the IPI, NCCN-IPI, and revised IPI are listed in Table 2. According to the IPI, NCCNIPI, and revised IPI stratification, nine, four, and 20 patients

Table I Patient characteristics

\begin{tabular}{ll}
\hline Patient characteristics & Value \\
\hline Number & 20 \\
Median age, years (range) & $59(30-75)$ \\
Male sex (\%) & 50 \\
IPI factors & \\
$\quad$ Age greater than 60 years (\%) & 65 \\
ECOG greater than 2 (\%) & 30 \\
Elevated LDH (\%) & 75 \\
More than I extranodal site (\%) & 75 \\
Stage III/IV (\%) & 75 \\
Tumor characteristics & \\
MYC overexpression (\%) & 65 \\
BCL-2 overexpression (\%) & 90 \\
MYC and BCL-2 overexpression (\%) & 60 \\
\hline Abbrevitions IPI Inter
\end{tabular}

Abbreviations: IPI, International Prognostic Index; ECOG, Eastern Cooperative Oncology Group. 
Table 2 Risk stratification and outcomes of 4-year OS and PFS

\begin{tabular}{|c|c|c|c|c|}
\hline Risk group & Number of prognostic factors & Patients & 4-yr PFS (\%) & 4-yr OS (\%) \\
\hline \multicolumn{5}{|l|}{$\mathrm{IPI}$} \\
\hline Low & 0,1 & 0 & - & - \\
\hline Low-intermediate & 2 & 0 & - & - \\
\hline High-intermediate & 3 & $1 \mathrm{I} / 20$ & 0 & 23.9 \\
\hline High & 4,5 & $9 / 20$ & 27.8 & 55.6 \\
\hline \multicolumn{5}{|l|}{ NCCN-IPI } \\
\hline Low & 0,1 & 0 & - & - \\
\hline Low-intermediate & 2,3 & $4 / 20$ & 0 & 0 \\
\hline High-intermediate & 4,5 & $12 / 20$ & 25.0 & 50.0 \\
\hline High & $6,7,8$ & $4 / 20$ & 0 & 50.0 \\
\hline \multicolumn{5}{|l|}{ Revised IPI } \\
\hline Low & 0 & 0 & - & - \\
\hline Intermediate & I, 2 & 0 & - & - \\
\hline High & $3,4,5$ & $20 / 20$ & 16.9 & 33.3 \\
\hline
\end{tabular}

Abbreviations: OS, overall survival; PFS, progression-free survival; IPI, International Prognostic Index; NCCN, National Comprehensive Cancer Network.

were classified as high risk, respectively. The risk groups demonstrated closely overlapping survival curves (Figures 1 and 2). Additionally, four out of 20 cases were identified as low-intermediate risk according to the NCCN-IPI criteria. The clinicopathological characteristics of the four patients are listed in Table 3.

\section{Discussion}

DLBCL remains a heterogeneous hematological disease with biologic indicators of treatment response and outcome that are not fully elucidated. ${ }^{1,2}$ The IPI was originally devised in the era before the introduction of rituximab. ${ }^{3}$ The use of rituximab has not only improved the survival of patients with DLBCL across all risk groups, ${ }^{4,5}$ but has also narrowed the prognostic differences between the IPI risk groups. ${ }^{6-8}$ Thus, a revision of the IPI for patients with DLBCL in the rituximab era using well-standardized clinical variables is needed.

Zhou et $\mathrm{a} \mathrm{I}^{19}$ presented a refined prognostic index (NCCNIPI) for patients with de novo DLBCL, using widely available clinical parameters. ${ }^{8}$ This model is straightforward and looks similar in many ways to the standard IPI. Zhou et al stated that the NCCN-IPI was easy to apply and more powerful than the standard IPI for predicting patients' prognosis in the rituximab era. ${ }^{19}$ Many hematologists have sought to identify new prognostic tools using molecular markers or gene expression, but none are ready to be broadly adopted into clinical practice. There has been a shift toward incorporating molecular marker profiling into prognostication. It is agreed that patients with DLBCL expressing both MYC and BCL-2 proteins have an aggressive clinical course and a poor
A

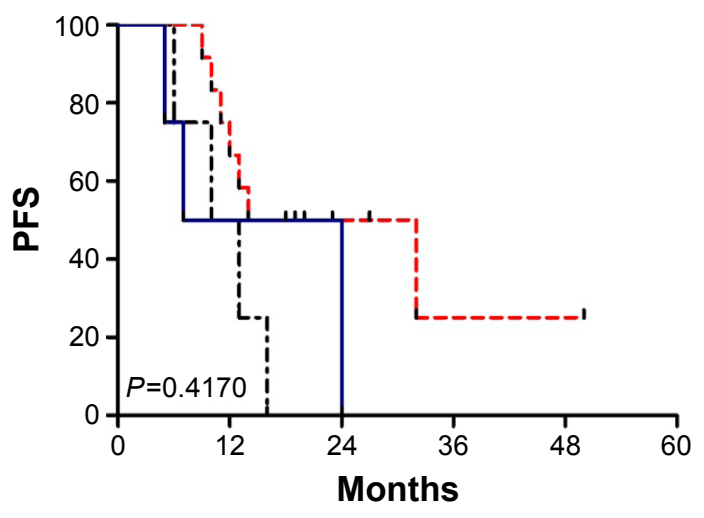

B

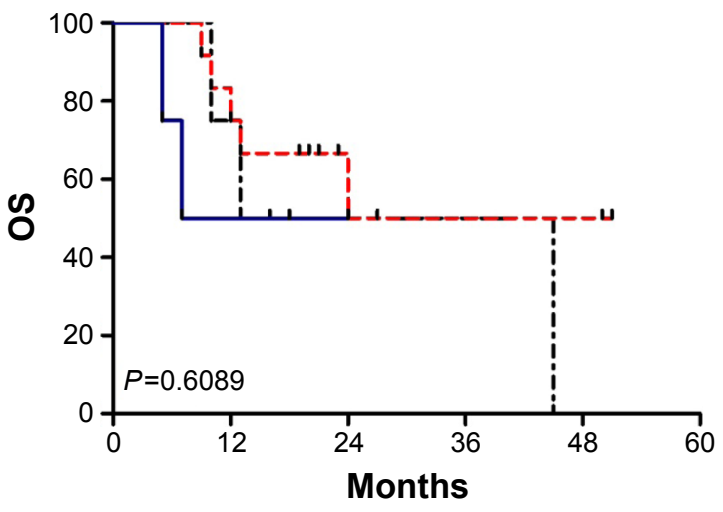

... Low-intermediate risk _L. High-intermediate risk ـ High risk

Figure I Survival according to the NCCN-IPI.

Notes: (A) Progression-free survival. (B) Overall survival.

Abbreviations: OS, overall survival; PFS, progression-free survival; IPI, International Prognostic Index; NCCN, National Comprehensive Cancer Network. 
A

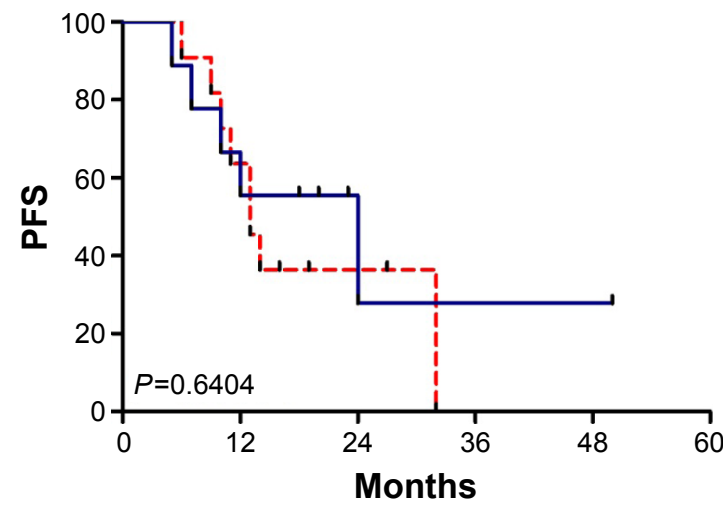

B

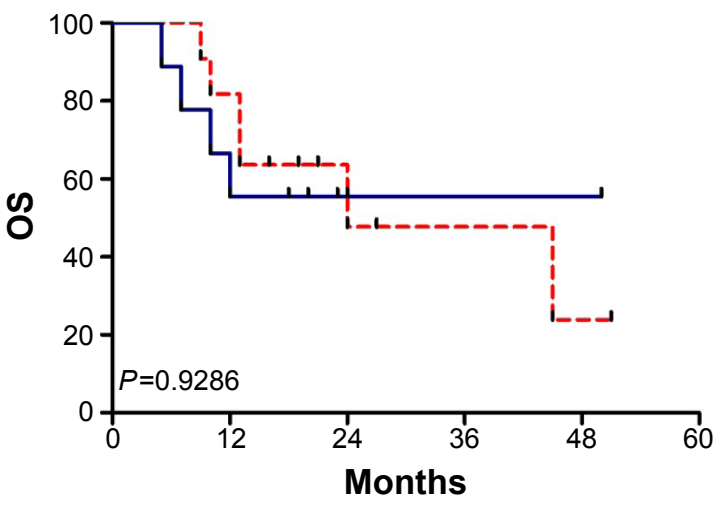

-ᄂ. High-intermediate risk $\perp$ High risk

Figure 2 Survival according to the IPI.

Notes: (A) Progression-free survival. (B) Overall survival.

Abbreviations: OS, overall survival; PFS, progression-free survival; IPI, International Prognostic Index; NCCN, National Comprehensive Cancer Network.

outcome. ${ }^{9,11}$ We have created a novel clinicopathologic prognostic model (biological marker-adjusted IPI, B-IPI) to identify a high-risk subgroup of DLBCL patients. On the basis of the number of negative prognostic factors present at the time of diagnosis, 20 patients were classified as high risk. These patients have high-risk clinicopathological features and poor outcomes. Was it an exaggerated prognosis to identify more high-risk patients in our study? Among these 20 high-risk patients, according to NCCN-IPI, four patients were assigned to the low-intermediate group. Three out of four patients were deceased within 16 months. Although one patient survived 45 months, his disease relapsed within 6 months. All four patients were found to overexpress both MYC and BCL-2 proteins. Obviously, these patients with a poor prognosis should be classified as high risk. In contrast to the IPI and NCCN-IPI, B-IPI has shown an improved capacity to discriminate high-risk patients. Our results were consistent with the revised-IPI. The revised-IPI was developed in a cohort of 365 patients. ${ }^{7}$ Our study showed that the revised-IPI and the B-IPI are substantially better for determining high-risk patients. High-risk groups have the most to gain from receiving novel agents as first-line therapy in place of less-effective conventional options, and these patients should be referred for clinical trials.

Eight predictors at diagnosis were identified in NCCNIPI, which looks similar to the standard IPI. It is generally agreed upon that patients with MYC/BCL-2 double-hit lymphomas (DHLs) have an extremely aggressive clinical course. Treatment is usually tailored according to the individual risk profile of a DLBCL patient. Petrich et $\mathrm{a}^{20}$ developed a novel risk score for DHL, which divides patients into high-, intermediate-, and low-risk groups. This DHL prognostic index resulted in excellent discrimination of a low-risk group with 2-year estimated OS rates of $91 \%$. Thus, elucidating a

Table 3 Four high-risk patients' characteristics

\begin{tabular}{lllll}
\hline & Patient number I & Patient number 2 & Patient number 3 & Patient number 4 \\
\hline Age (years) & 50 & 30 & $5 \mathrm{I}$ & 53 \\
Sex & Male & Male & Male & Male \\
ECOG & $\mathrm{I}$ & $\mathrm{I}$ & $\mathrm{I}$ & $\mathrm{I}$ \\
LDH (220 U/L) & 493 & 404 & 350 & 107 \\
Extranodal site of disease & - & Bone marrow & - & Bone marrow \\
Stage & $\mathrm{III}$ & $\mathrm{IV}$ & $\mathrm{III}$ & $\mathrm{IV}$ \\
MYC expression (\%) & 95 & 65 & 85 & 60 \\
BCL-2 expression (\%) & 85 & 85 & 75 & 50 \\
Replase (months) & 6 & 12 & 10 & 15 \\
Survival (months) & 45 & 13 & 10 & 16 \\
NCCN-IPI score & 3 & 3 & 3 & 3 \\
\hline
\end{tabular}

Abbreviations: IPI, International Prognostic Index; ECOG, Eastern Cooperative Oncology Group; LDH, lactate dehydrogenase; NCCN, National Comprehensive Cancer Network. 
better predictor of prognosis without consideration of these biological markers would be inappropriate. The addition of the expression of MYC and BCL-2 proteins to the IPI would be helpful in identifying patients with DHL who carry a particularly favorable prognosis. Immunohistochemistry is a rapid and inexpensive method for detecting these biological markers. Immunohistochemistry can be easily applied in academic and community-based hospitals. While our study is a retrospective study with a small number of patients, whether the B-IPI can serve as a prognostic guide for risk-adapted therapy remains to be seen and will depend on whether our results can be confirmed in prospective trials.

In summary, biological marker-adjusted IPI could identify a subset of DLBCL patients with high-risk clinicopathological features and poor clinical outcome.

\section{Acknowledgments of grant support}

Medical Science and Technology Key Project of Nanjing, NJGL-2011196. Jiangsu Provincial Special Program of Medical Science, BL2012005. Medical Science and Technology Project of Nanjing, ZKX10012. Medical Science and Technology Key Project of Nanjing, ZKX14015. Peak of Six Talent in Jiangsu Province, 2014-WSN-049.

\section{Author contributions}

All authors contributed toward data analysis, drafting and revising the paper and agree to be accountable for all aspects of the work.

\section{Disclosure}

The authors report no conflicts of interest in this work.

\section{References}

1. Swerdlow SH, Campo E, Harris NL, et al. WHO Classification of Tumours of Haematopoietic and Lymphoid Tissues. Geneva, Switzerland: WHO Press; 2008.

2. Smith A, Roman E, Howell D, et al. The Haematological Malignancy Research Network (HMRN): a new information strategy for population based epidemiology and health service research. Br J Haematol. 2010; 148(5):739-753.

3. Project TIN-HsLPF. A predictive model for aggressive non-Hodgkin's lymphoma. The International Non-Hodgkin's Lymphoma Prognostic Factors Project. N Engl J Med. 1993;329(14):987-994.

4. Feugier P, Van Hoof A, Sebban C, et al. CHOP chemotherapy plus rituximab compared with $\mathrm{CHOP}$ alone in elderly patients with diffuse large-B-cell lymphoma. $N$ Engl J Med. 2002;346(4):235-242.
5. Hauptrock B, Hess G. Rituximab in the treatment of non-Hodgkin's lymphoma. Biologics. 2008;2(4):619-633.

6. Sehn LH. Paramount prognostic factors that guide therapeutic strategies in diffuse large B-cell lymphoma. Hematology Am Soc Hematol Educ Program. 2012;2012:402-409.

7. Sehn LH, Berry B, Chhanabhai M, et al. The revised International Prognostic Index (R-IPI) is a better predictor of outcome than the standard IPI for patients with diffuse large B-cell lymphoma treated with R-CHOP. Blood. 2007;109(5):1857-1861.

8. Sengar M, Menon H, Dangi $\mathrm{H}$, et al. Comparison of treatment outcomes with EPOCH-Rituximab versus CHOP-Rituximab in patients with denovo intermediate and high risk international prognostic index (IPI) diffuse large B cell lymphoma (DLBCL): a single center retrospective analysis. Blood. 2013;122:e5115.

9. Xia B, Zhang L, Guo SQ, et al. Coexpression of MYC and BCL-2 predicts prognosis in primary gastrointestinal diffuse large B-cell lymphoma. World J Gastroenterol. 2015;21(8):2433-2442.

10. Zhou M, Wang J, Ouyang J, et al. MYC protein expression is associated with poor prognosis in diffuse large B cell lymphoma patients treated with RCHOP chemotherapy. Tumour Biol. 2014;35(7):6757-6762.

11. Perry AM, Alvarado-Bernal Y, Laurini JA, et al. MYC and BCL2 protein expression predicts survival in patients with diffuse large B-cell lymphoma treated with rituximab. Br J Haematol. 2014;165(3):382-391.

12. Kluk MJ, Chapuy B, Sinha $P$, et al. Immunohistochemical detection of MYC-driven diffuse large B-cell lymphomas. PLoS One. 2012;7(4): e33813.

13. Tapia G, Lopez R, Muñoz-Mármol AM, et al. Immunohistochemical detection of MYC protein correlates with MYC gene status in aggressive B cell lymphomas. Histopathology. 2011;59(4):672-678.

14. Maeshima AM, Taniguchi H, Fukuhara S, et al. Bcl-2, Bcl-6, and the International Prognostic Index are prognostic indicators in patients with diffuse large B-cell lymphoma treated with rituximab-containing chemotherapy. Cancer Sci. 2012;103(10):1898-1904.

15. Green TM, Young KH, Visco C, et al. Immunohistochemical double-hit score is a strong predictor of outcome in patients with diffuse large B-cell lymphoma treated with rituximab plus cyclophosphamide, doxorubicin, vincristine, and prednisone. J Clin Oncol. 2012;30(28):3460-3467.

16. Hu S, Xu-Monette ZY, Tzankov A, et al. MYC/BCL2 protein coexpression contributes to the inferior survival of activated B-cell subtype of diffuse large B-cell lymphoma and demonstrates high-risk gene expression signatures: a report from the International DLBCL Rituximab-CHOP Consortium Program. Blood. 2013;121(20):4021-4031.

17. Johnson NA, Slack GW, Savage KJ, et al. Concurrent expression of MYC and BCL-2 in diffuse large B-cell lymphoma treated with rituximab plus cyclophosphamide, doxorubicin, vincristine, and prednisone. J Clin Oncol. 2012;30(28):3452-3459.

18. Wang J, Zhou M, Xu JY, et al. MYC and BCL-2 adjusted-International Prognostic Index (A-IPI) is a better predictor of outcome than the standard IPI for patients with diffuse large B-cell lymphoma treated with R-CHOP. Histol Histopathol. In press 2015.

19. Zhou Z, Sehn LH, Rademaker AW, et al. An enhanced International Prognostic Index (NCCN-IPI) for patients with diffuse large B-cell lymphoma treated in the rituximab era. Blood. 2014;123(6):837-842.

20. Petrich AM, Gandhi M, Jovanovic B, et al. Impact of induction regimen and stem cell transplantation on outcomes in double-hit lymphoma: a multicenter retrospective analysis. Blood. 2014;124(15):2354-2361. 


\section{Supplementary material}
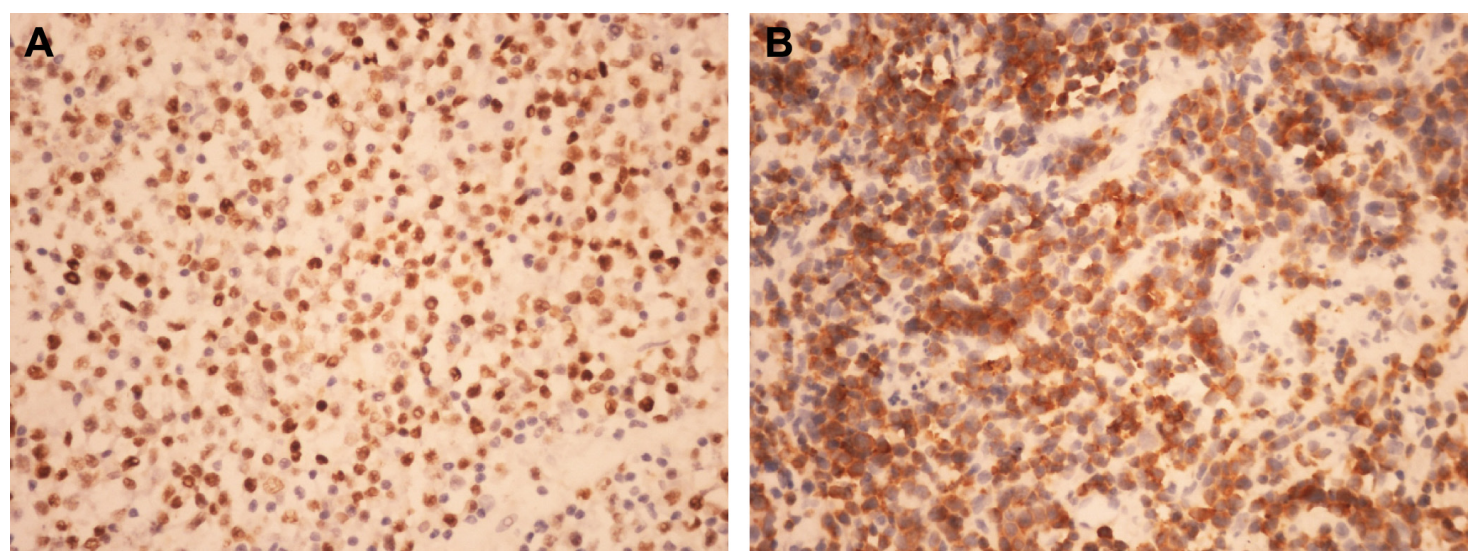

Figure SI (A) MYC protein expression was exclusively brown nuclear ( $\times 400)$. (B) BCL-2 protein expression was exclusively brown cytoplasm $(\times 400)$.

\section{Publish your work in this journal}

OncoTargets and Therapy is an international, peer-reviewed, open access journal focusing on the pathological basis of all cancers, potential targets for therapy and treatment protocols employed to improve the management of cancer patients. The journal also focuses on the impact of management programs and new therapeutic agents and protocols on patient perspectives such as quality of life, adherence and satisfaction. The manuscript management system is completely online and includes a very quick and fair peer-review system, which is all easy to use. Visit http://www.dovepress.com/testimonials.php to read real quotes from published authors. 